\title{
Personalized management of cirrhosis by non-invasive tests of liver fibrosis
}

\author{
Grace Lai-Hung Wong ${ }^{1,2}$, Wendell Zaragoza Espinosa', and Vincent Wai-Sun Wong ${ }^{1,2}$ \\ 'Department of Medicine and Therapeutics and 'State Key Laboratory of Digestive Disease, The Chinese University of Hong Kong, \\ Hong Kong
}

Owing to the high prevalence of various chronic liver diseases, cirrhosis is one of the leading causes of morbidity and mortality worldwide. In recent years, the development of non-invasive tests of fibrosis allows accurate diagnosis of cirrhosis and reduces the need for liver biopsy. In this review, we discuss the application of these non-invasive tests beyond the diagnosis of cirrhosis. In particular, their role in the selection of patients for hepatocellular carcinoma surveillance and varices screening is highlighted. (Clin Mol Hepatol 2015;21:200-211)

Keywords: Transient elastography; FibroScan; FibroTest; Hepatocellular carcinoma; Varices

\section{INTRODUCTION}

Despite geographical differences, chronic liver diseases are highly prevalent worldwide. It is estimated that at least 350 million and 120 million people globally are chronically infected with hepatitis B virus (HBV) and hepatitis C virus, respectively. ${ }^{1,2}$ Nonalcoholic fatty liver disease (NAFLD) affects $15-40 \%$ of the general population and is particularly prevalent in patients with diabetes and obesity. ${ }^{3-5}$ Alcoholic liver disease affects both developed and developing countries and may account for up to $9.2 \%$ of all disability-adjusted life years in some regions. ${ }^{6}$ Although the etiologies are different, chronic liver disease leads to liver injury, progressive liver fibrosis, and finally to the stage of cirrhosis and liver decompensation. As a result, cirrhosis remains the twelfth global leading cause of death in 2010 .

The diagnosis of cirrhosis is not as simple as it seems. Evidently, the diagnosis is straightforward when a patient has already devel- oped clinical manifestations of portal hypertension such as ascites, varices and hypersplenism. Nonetheless, these signs are absent in patients with early cirrhosis, and the radiological features of early cirrhosis are subtle and unreliable. ${ }^{8}$ Liver biopsy is traditionally the gold standard for the diagnosis of cirrhosis. However, it is an invasive procedure with a small risk of bleeding. The poor patient acceptance and the risk of sampling error (understaging due to inadequate sample) further limit the widespread application of liver biopsy.

In recent years, the development and application of non-invasive tests of liver fibrosis have revolutionized hepatology practice. Numerous studies have confirmed the accuracy of these tests in fibrosis staging and the diagnosis of cirrhosis. In general, the tests have high negative predictive value in excluding advanced fibrosis and cirrhosis and have been recommended by the European Association for the Study of the Liver as initial assessment in patients with various liver diseases. ${ }^{9}$

\footnotetext{
Abbreviations:

ALT, alanine aminotransferase; APRI, AST-to-platelet ratio index: AST, aspartate aminotransferase; CHB, chronic hepatitis B; CHC, chronic hepatitis C; EGD,esophagogastroduodenoscopy; ELF, enhanced liver fibrosis; HBV, hepatitis B virus; HCC, hepatocellular carcinoma; $\mathrm{HCV}$, hepatitis $\mathrm{C}$ virus; HR, hazard ratio; HVPG, hepatic vein pressure gradient; LSM, liver stiffness measurement; NAFLD, non-alcoholic fatty liver disease
}

\author{
Corresponding author: Vincent Wai-Sun Wong \\ Department of Medicine and Therapeutics and State Key Laboratory \\ of Digestive Disease, The Chinese University of Hong Kong, 9/F, Clinical \\ Sciences Building, Prince of Wales Hospital, 30-32 Ngan Shing Street, \\ Shatin, Hong Kong \\ Tel: +852 26323942, Fax: +852 26373852 \\ E-mail:wongv@cuhk.edu.hk
}


In addition, cirrhosis is not one single disease but encompasses a broad spectrum of clinical condition ranging from compensated disease to decompensated disease. As the disease progresses, various complications of portal hypertension may develop. The development of hepatocellular carcinoma (HCC) further drifts the clinical course and leads to major morbidity and mortality. Therefore, one important part of the management of cirrhosis is to identify and treat major complications early. In this review, we first provide an overview on non-invasive tests of liver fibrosis. Since the diagnosis of cirrhosis is only the first step in the management of cirrhosis, we further discuss the potential application of these tests in the risk stratification of cirrhosis and prediction of cirrhotic complications.

\section{NON-INVASIVE TESTS OF LIVER FIBROSIS}

Non-invasive tests of liver fibrosis have been a hot research area in the past decade. At the beginning, the main focus was to reduce the burden of liver biopsy by confidently identifying patients who are very unlikely to have significant fibrosis on one hand and those who are very likely to have advanced fibrosis or cirrhosis on the other. Treatment decisions can then be made accordingly, and patients in the middle (gray zone cases) may undergo liver biopsy or be observed over time. In general, non-invasive tests of liver fibrosis can be divided into serum tests and physical measurements.

\section{Serum tests}

The advantages of serum tests include high applicability (successful measurements can be made in most cases) and relatively simple logistics. Doctors may obtain blood samples at their clinics and send them to designated laboratories even for more specific biomarkers. Serum tests can be divided into class I biomarkers and class II biomarkers. Class I biomarkers specifically measure the activity of fibrogenesis or fibrinolysis. In contrast, class II biomarkers do not measure fibrosis directly but represent parameters that correlate with fibrosis. For example, aspartate aminotransferase (AST) is a marker of hepatic necroinflammation and not fibrosis. However, patients with fibrosis and cirrhosis often have increased AST levels. Although class I biomarkers are expected to directly reflect fibrosis and be more accurate than class II biomarkers, this has not been consistently demonstrated in prospective studies.

In any case, at present there is no single marker that can ade- quately reflect fibrosis. Therefore, in most situations several biomarkers or a combination of biomarkers and other clinical features are used. Some of the combined panels such as FibroTest, FibroMeter and enhanced liver fibrosis (ELF) score have been commercialized. It should be noted that such combined tests are modeled against liver histology, which is an imperfect reference standard. In other words, even if the models can 100\% faithfully reflect liver histology, the accuracy of liver histology to diagnose fibrosis and cirrhosis will be the ceiling of accuracy of the new models. ${ }^{10}$

Some of the class II biomarkers are more generic. Examples include the AST-to-alanine aminotransferase (ALT) ratio and the AST-to-platelet ratio index (APRI). In other cases, owing to the pathophysiology of different liver diseases, the class II biomarkers are more disease-specific. For instance, metabolic factors are overrepresented in the NAFLD fibrosis score, which should only be applied in patients with NAFLD. ${ }^{11,12}$

\section{Physical measurements}

The other main class of non-invasive tests of liver fibrosis relies on physical measurement of liver stiffness and elasticity. Although the cutoffs of the measurements are determined with reference to histology, these tests are not modeled against histology and theoretically may achieve better prediction than histology. In fact, studies in patients with chronic viral hepatitis suggest that transient elastography may be better than histology in predicting overall mortality. ${ }^{13,14}$

Transient elastography by FibroScan (Echosens, Paris, France) is currently the most commonly used method to measure liver stiffness. ${ }^{15}$ It estimates liver fibrosis by measuring the velocity of a shear wave in the liver parenchyma. This is based on the physical principle that waves travel faster in a stiffer medium. The main advantage of transient elastography is the ease of use and high reproducibility. ${ }^{16}$ Compared with serum tests, transient elastography is less applicable in obese patients, although the new $\mathrm{XL}$ probe partially compensates for that. ${ }^{17}$ Liver stiffness is also affected by high ALT level, hepatic congestion, food intake and amyloidosis. Because the correlation between liver stiffness and fibrosis is a generic phenomenon, the technique can be applied to patients with different liver diseases, though the appropriate cutoffs are higher in patients with alcoholic liver disease. ${ }^{18}$

Newer techniques such as acoustic radiation force impulse and shear-wave elastography allow simultaneous visualization of the liver parenchyma and measurement of liver elasticity. ${ }^{19,20}$ This can thus combine HCC surveillance and liver fibrosis assessment in a single examination. Magnetic resonance elastography appears to 
be highly accurate and is not affected by obesity, but machine availability and the costs of examination can be prohibitive. ${ }^{21}$ These new techniques also have not been as extensively validated as transient elastography.

For simplicity, doctors usually adopt one or more recommended cutoff values in the interpretation of any of the non-invasive tests above. In reality, however, the more extreme the values of the non-invasive tests are, the more we are confident in whether a patient has fibrosis/cirrhosis or not. For example, a probabilitybased interpretation of liver stiffness measurements (LSM) has been proposed. ${ }^{22}$ Based on the exact LSM, the probability of a patient having different fibrosis stages can be determined. The complexity of this approach limits its use in real life practice. Nonetheless, as a patient is more likely to have cirrhosis and advanced cirrhosis when he has more extreme non-invasive tests results, this forms the basis of extending the tests to stratify the risk and predict complications in cirrhotic patients.

\section{HEPATOCELLULAR CARCINOMA}

\section{Current recommendations and unmet need}

HCC is one of the most important complications in patients with chronic liver diseases. ${ }^{23} \mathrm{HCC}$ surveillance is an indispensable part of the management of liver patients. The current Asian Pacific guidelines recommend 6-monthly trans-abdominal ultrasonography and serum alpha-fetoprotein (AFP) testing for HCC surveillance. ${ }^{24}$ Despite the fact that AFP has been widely adopted for decades, it has been criticized as neither sensitive nor specific. ${ }^{25}$ Hence the latest American guidelines stopped recommending the use of AFP in the surveillance program. ${ }^{26}$ Nonetheless, a recent study demonstrated a satisfactory sensitivity and specificity of AFP for HCC in CHB patients received antiviral therapy. ${ }^{27}$ Therefore some experts believed that the calls for abandoning the monitoring of AFP levels might be premature, especially given the already low HCC surveillance rate in developing countries and at primary care settings. ${ }^{28}$

HCC surveillance improves the prognosis of patients by identifying tumors of smaller sizes, fewer numbers of tumors, and longer overall survival. ${ }^{29}$ Unfortunately, the 5 -year mortality rate was still close to $40 \%$ despite the regular HCC surveillance. This observation points towards the fact that the current HCC surveillance recommendation is still far from satisfactory.

The key components of an optimal surveillance program include accurate risk stratification and reliable surveillance tools. Hence there is a need for accurate $\mathrm{HCC}$ risk prediction to assist prognostication as well as decision on the need for HCC surveillance.

\section{Non-invasive tests and HCC risk}

\section{Liver stiffness measurements}

Various non-invasive tests of liver fibrosis have been tested to predict the risk of HCC. Among them LSM with transient elastography is the most widely-studied. A dose-response relationship between LSM and HCC risk was demonstrated in patients with either chronic hepatitis B (CHB) or chronic hepatitis C (CHC). ${ }^{30,31}$ In a prospective cohort of study of 1,130 Korean CHB patients, the hazard ratios (HRs) of developing HCC were 3.1, 4.7, 5.6, and 6.6 in patients with LSM at 8.1-13.0 $\mathrm{kPa}, 13.1-18.0 \mathrm{kPa}, 18.1-23.0$ $\mathrm{kPa}$, and $>23.0 \mathrm{kPa}$, respectively, when compared to those with $L S M L S M \leq 8.0 \mathrm{kPa} .{ }^{30} \mathrm{~A}$ similar relationship was demonstrated in

Table 1. CU-HCC score vs. LSM-HCC score [Modified from reference Wong VW et al. JCO 2010 \& Wong GL et al. J Hep 2014]

\begin{tabular}{lcc}
\hline Factors & CU-HCC score & LSM-HCC score \\
\hline Age & & \\
$>50$ years & +3 & +10 \\
$\leq 50$ years & 0 & 0 \\
Albumin & & \\
$\leq 35 \mathrm{~g} / \mathrm{L}$ & +20 & +1 \\
$>35 \mathrm{~g} / \mathrm{L}$ & 0 & 0 \\
Total bilirubin $(\mu \mathrm{mol} / \mathrm{L})$ & & \\
$>18$ & +1.5 & \\
$\leq 18$ & 0 & \\
HBV DNA & & +5 \\
$>200,000 \mathrm{IU} / \mathrm{mL}$ & +4 & 0 \\
$2,000-200,000 \mathrm{IU} / \mathrm{mL}$ & +1 & \\
$\leq 2,000 \mathrm{IU} / \mathrm{mL}$ & 0 & \\
Cirrhosis & & \\
Yes & +15 & \\
No & 0 & \\
\hline
\end{tabular}

Liver stiffness measurement

$\begin{array}{lc}\leq 8.0 \mathrm{kPa} & 0 \\ 8.1-12.0 \mathrm{kPa} & +8 \\ >12.0 \mathrm{kPa} & +14\end{array}$

HBV, hepatitis B virus; LSM, liver stiffness measurement.

${ }^{*}$ Total CU-HCC score ranges from 0 to 44.5. Scores of 0 to 4, 5 to 19 and 20 to 44.5 indicate low, intermediate and high risk respectively.

${ }^{\dagger}$ Total LSM-HCC score ranges from 0 to 30 . Scores of 0 to 10,11 to 20 and 21 to 30 indicate low, intermediate and high risk respectively. 
Grace Lai-Hung Wong, et al.

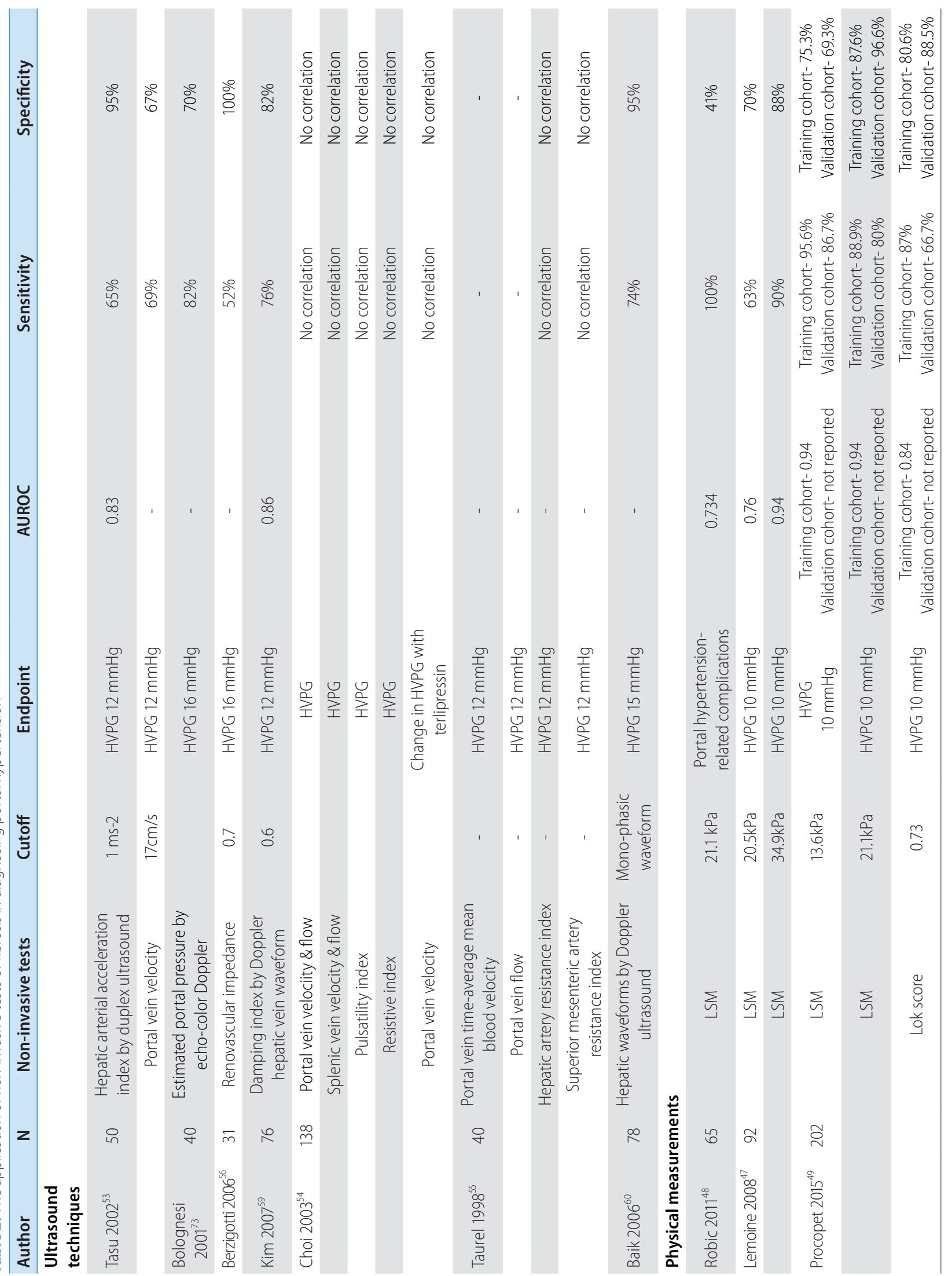




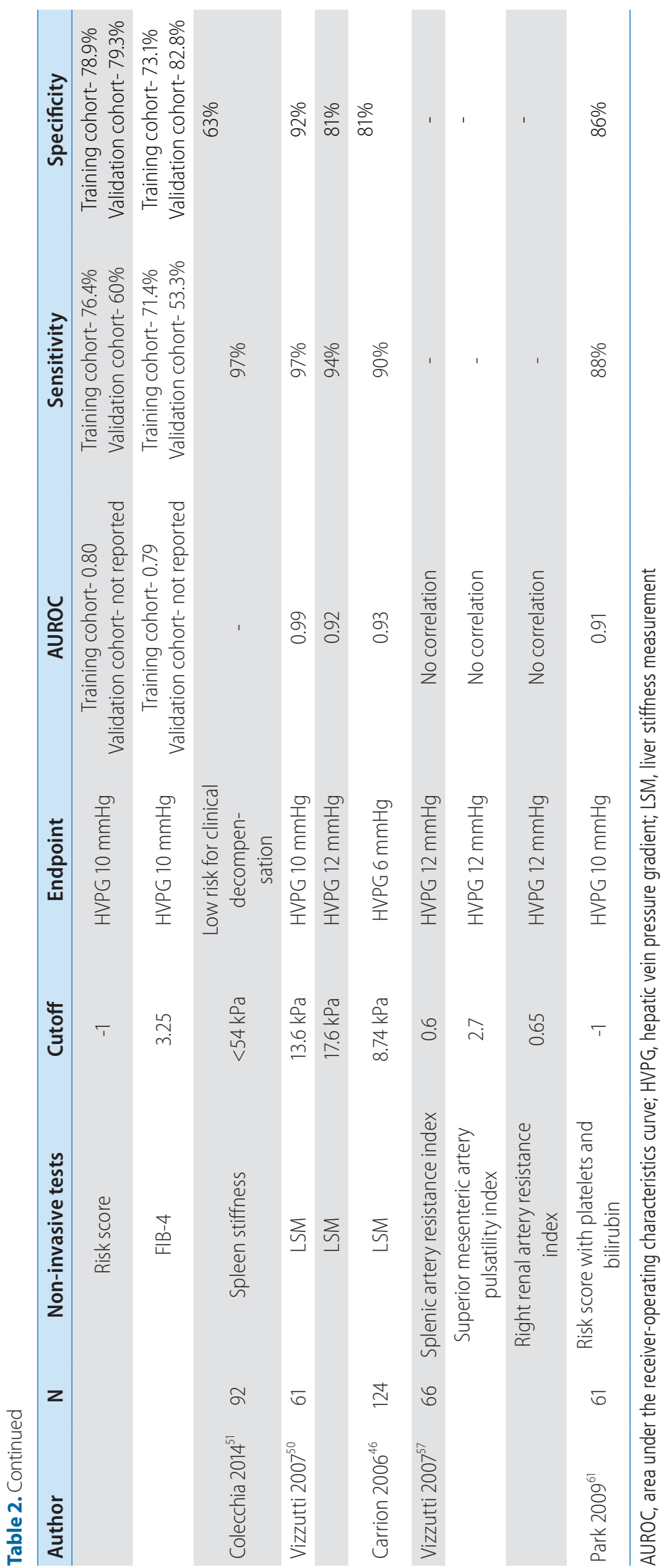

another prospective cohort of 866 Japanese CHC patients that the HRs of HCC were 17, 21, 26, and 46 in patients with LSM at 10.1-15.0 $\mathrm{KPa}, 15.1-20.0 \mathrm{kPa}, 20.1-25.0 \mathrm{kPa}$, and $>25.0 \mathrm{kPa}$, respectively, with reference to those with LSM $\leq 10.0 \mathrm{kPa} .^{31}$ These findings implied that LSM is a useful parameter to estimate $\mathrm{HCC}$ risk in patients with chronic liver disease across the etiologies despite different carcinogenetic mechanisms.

\section{Serum tests}

FibroTest is one of most popular serum-based non-invasive tests for liver fibrosis. ${ }^{32}$ FibroTest was recently shown to be as good as LSM to stage patients with viral hepatitis into seven categories such that it could accurately predict severe complications, of which most were HCC. ${ }^{33,34}$ In a multicenter study of 1,312 French patients with chronic hepatitis $B$, using the predetermined stages of FibroTest at $\leq 0.27,>0.27-0.48,>0.48-0.58,>0.58-0.74,>0.74-$ 0.85 and $>0.85-1$, HCC developed in $0.4 \%, 0.7 \%, 4.4 \%$, $13.6 \%, 11.1 \%$ and $44.7 \%$ of patients, respectively. ${ }^{34}$

Another serum-based non-invasive test, enhanced liver fibrosis (ELF) test has been increasingly used to assess liver fibrosis by its or in combination with LSM in CHB patients. ${ }^{35}$ In a Korean study, ELF was found superior to LSM, histologic fibrosis stage, or age-spleen-platelet ratio to predict liver-related events, again HCC accounting for most of the events. ${ }^{36}$ Compared with patients with high ELF $\geq 10.40$, patients of low $(<8.10)$ and intermediate (8.10-10.39) ELF scores had much lower risk of liver-related events (adjusted HR 0.045 and 0.239 respectively). ${ }^{36}$

\section{$\mathrm{HCC}$ risk score based on non-invasive tests}

The risk factors of HCC in patients with chronic hepatitis $B$ are well known, and various groups have derived HCC risk scores based on the factors. ${ }^{37}$ Since cirrhosis is the single most important risk factor for HCC development, noninvasive tests of fibrosis may improve prediction by not only diagnosing cirrhosis more accurately but also reflecting the severity of cirrhosis. To further consolidate the important role of LSM on HCC risk prediction, it was incorporated into a risk score for HBV-related HCC, called LSM-HCC score, together with three other important clinical parameters namely age, serum albumin and HBV DNA level. ${ }^{38}$ LSM has replaced clinical cirrhosis, the heavily weighed component in the original CU-HCC score, ${ }^{39}$ in order to provide more 


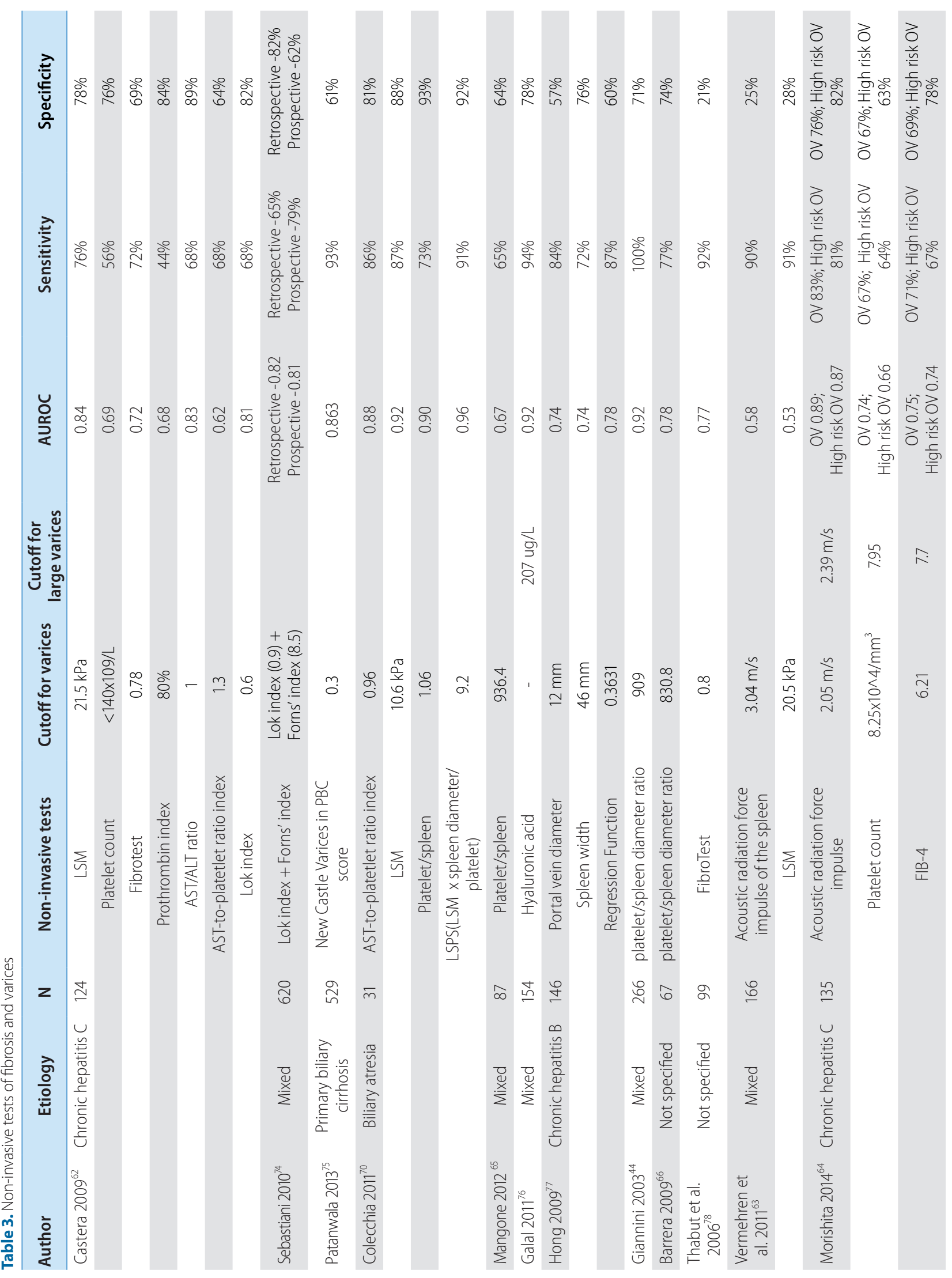




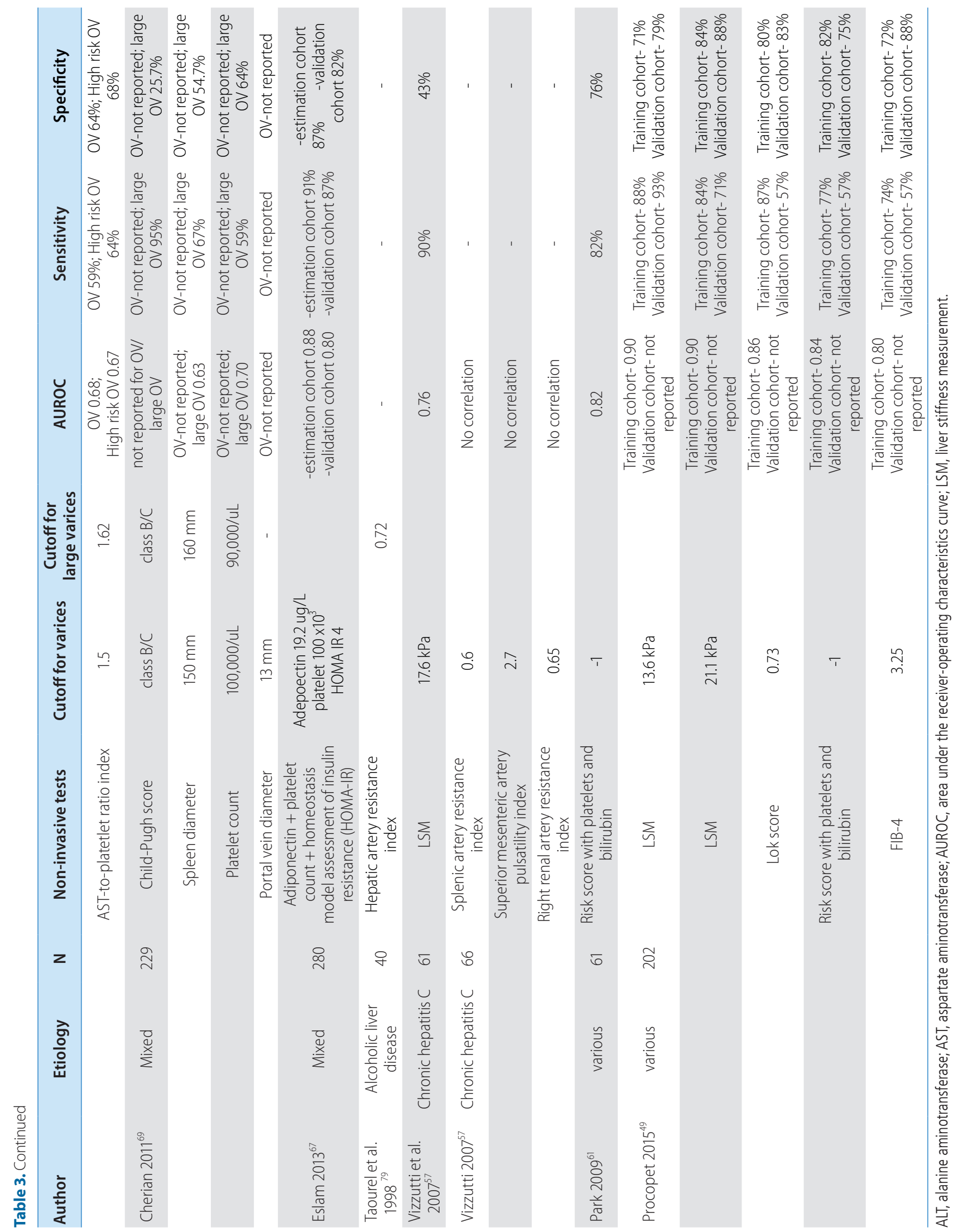


objective and accurate diagnosis of cirrhosis (Table 1). This new LSM-HCC score excludes future HCC with high negative predictive value $(99.4-100 \%)$ at 5 years. ${ }^{38}$

LSM has also been integrated with age, gender, HBV DNA level into a regression formula to predict HCC with good accuracy. ${ }^{40}$ Adding LSM to another commonly used HCC risk score REACH-B score achieved a higher accuracy to predict liver-related events (of which $60 \%$ were HCC) when compared to REACH-B score alone (area under the receiver-operating characteristics curve 0.81 vs. 0.63). ${ }^{41}$ Although serum-based non-invasive tests are probably equally popular for assessing liver fibrosis, so far no HCC risk score has been developed based on these tests.

\section{VARICES}

\section{Current recommendations and unmet need}

Portal hypertension is one of the most lethal complications of chronic liver disease. ${ }^{42}$ Thus, it is vital to identify patients with varices and institute primary prophylaxis. According to the American Association for the Study of Liver Diseases, all diagnosed patients with cirrhosis should undergo screening esophagogastroduodenoscopy (EGD). ${ }^{43}$ For patients without varices during screening, surveillance EGD should be done after two to three years. For those with small varices, surveillance EGD is recommended after one to two years. Annual EGD is warranted in patients with liver decompensation. ${ }^{43}$

However, EGD is unpleasant. Some patients at risk would never agree to undergo $E G D$ because of the perceived discomfort. On the other hand, many other patients go through the procedure with negative findings. This may cause unnecessary suffering and increase health costs. The use of thrombocytopenia and splenomegaly has been proposed to identify patients with portal hypertension and varices, but the accuracy remains limited. ${ }^{44}$

\section{Non-invasive tests and portal hypertension}

Ascites, hepatic encepalopathy, and variceal bleeding are some of the complications of liver cirrhosis. The development of these events is due to portal hypertension. The gold standard for detecting portal hypertension is through the measurement of hepatic venous pressure gradient (HVPG). However, it is invasive and not readily available. Therefore, a number of studies have evaluated the use of non-invasive tests to predict portal hypertension (Table 2). One of the most commonly used is transient elastography. Given that fibrosis is a major contributor to elevated hepatic resistance (measured by HVPG), the role of transient elastography as a surrogate measure of HVPG has been studied. ${ }^{45}$ Several cutoff levels have been proposed ranging from $8.7 \mathrm{kPa}$ (HVPG $>6$ $\mathrm{mmHg}$ ) to $34.9 \mathrm{kPa}$ (HVPG >10 mmHg) depending on the population being tested. ${ }^{46,47}$ It has a sensitivity of $63-100 \%$ and a specificity of $41-96 \%$ to determine portal hypertension. ${ }^{46-50}$ In addition, in a study among cirrhotics, an LSM of $>21.1 \mathrm{kPa}$ is as accurate as HVPG in identifying patients at risk portal hypertensive complications. ${ }^{48}$

Splenomegaly and hypersplenism are features of portal hypertension. Indeed, spleen stiffness (54 kPa) can predict survival free complications among HCV-related cirrhotic patients. ${ }^{48,51}$ However, it should be highlighted that transient elastography measures a core of tissue that is $4 \mathrm{~cm}$ in length; it has not been optimized for the measurement of spleen stiffness. In that regard, other physical measurements such as acoustic radiation force impulse may be more appropriate. ${ }^{52}$

Furthermore, the use of duplex doppler ultrasonography has been an attractive non-invasive test to determine portal hypertension by assessing the vascular anatomy and its hemodynamics. Parameters such as portal blood flow and velocity, resistive and pulsatility indices have also been explored to predict HVPG although with conflicting results. In a study of cirrhotic patients with different etiologies, portal vein velocity was found to correlate with HVPG, but the findings have not been confirmed by all studies. ${ }^{53-55}$ Other examinations of the hepatic, splenic, superior mesenteric arteries were not proven to be correlated with HVPG. ${ }^{53-58}$ Interestingly, some ultrasound parameters such as hepatic waveforms and damping index may be used to determine the response to medications to decrease portal pressure. ${ }^{59,60}$ In contrast, portal vein velocity is not useful for the monitoring of response to terlipressin. ${ }^{54,58}$ These conflicting results may be due to differences in techniques used and heterogeneity of populations being studied such as of etiology of cirrhosis, Child Pugh score, and use of medications.

A Korean group further developed a simple risk score comprising bilirubin and platelets to predict HVPG. ${ }^{61}$ It has a sensitivity of up to $88 \%$ and specificity of up to $86 \%$ in predicting HVPG $>10$ $\mathrm{mmHg}$. Other tests like the Lok index and FIB-4 had fair accuracy in predicting $\mathrm{HVPG} .{ }^{49,61}$

\section{Non-invasive tests and varices}

As mentioned above, EGD should be performed for varices screening in cirrhotic patients, but the procedure is unpleasant and often omitted. Since non-invasive tests of fibrosis can reflect 
portal hypertension, it is logical to consider their application in selecting patients for EGD. LSM has the highest accuracy with a sensitivity of $76-91 \%$ and specificity varies of $28-88 \%$ to detect esophageal varices (Table 3). ${ }^{50,51,62,63}$ Some authors used acoustic radiation force impulse of spleen and liver with good accuracy (ARFI spleen sensitivity 90\% and specificity 76\%; ARFI liver sensitivity $83 \%$ and specificity $76 \%)^{63,64}$ Several studies have evaluated the platelet-to-spleen ratio with an adequate sensitivity of 64$100 \%$ and specificity $64-93 \%{ }^{44,51,65,66}$ Furthermore, to predict high risk varices or variceal bleeding, ARFI and Child-Pugh class were noted to correlate with these end points. ${ }^{64,67}$ Other blood tests that were determined to predict varices were platelet count (sensitivity 56-76\%; specificity 64-88\%) and AST-to-platelet Index (sensitivity 59-86\%; specificity $64-81 \%$ ). ${ }^{62,64,68-70}$ Among the doppler ultrasound parameters only hepatic artery resistive index was found to be associated with presence of varices. ${ }^{55,57}$

In the recent Baveno VI guideline, some of these non-invasive tests were already incorporated to stratify patients..$^{71}$ It recommends that patients with $\mathrm{LSM}<20 \mathrm{kPa}$ and platelet count $>$ 150,000 have very low risk of having large varices; screening EGD can be avoided. However, these very low risk patients should undergo annual transient elastography and platelet count.

\section{CONCLUSIONS}

Non-invasive tests of liver fibrosis have revolutionized the management of chronic liver diseases. Compared with routine clinical assessments, the non-invasive tests allow more confident diagnosis of cirrhosis and can also reflect the severity of cirrhosis and/or portal hypertension. They can therefore be used to select patients for HCC surveillance and varices screening.

That said, there are still a number of questions regarding the use of the non-invasive tests. The interval of testing is currently undefined, and it is unclear to what extent the changes in the non-invasive tests reflect fibrosis progression. In addition, patients with treated viral hepatitis often have reduced liver stiffness and improved serum tests of fibrosis. ${ }^{72}$ While some of these patients indeed have reduced fibrosis, it is unclear when this indicates regression of cirrhosis and whether the interpretation is the same as in untreated patients. Similarly, non-selective beta-blockers reduce portal pressure and are the firstline treatment in patients with varices. Whether physical measurements of liver and spleen stiffness can reflect the response to beta-blockers remains unclear. The non-invasive tests can be used more appropriately when such longitudinal data become available.

\section{Conflicts of Interest}

Grace Wong has served as an advisory board member for Gilead and received paid lecture fees from AbbVie, Bristol-Myers Squibb, Echosens, Gilead and Janssen. Vincent Wong has served as an advisory board member for Gilead and Janssen and a consultant for AbbVie, Merck and NovoMedica, and received paid lecture fees from AbbVie, Echosens and Gilead.

\section{REFERENCES}

1. Trépo C, Chan HL, Lok A. Hepatitis B virus infection. Lancet 2014;384:2053-2063.

2. Shepard CW, Finelli L, Alter MJ. Global epidemiology of hepatitis C virus infection. Lancet Infect Dis 2005;5:558-567.

3. Williams CD, Stengel J, Asike MI, Torres DM, Shaw J, Contreras M, et al. Prevalence of nonalcoholic fatty liver disease and nonalcoholic steatohepatitis among a largely middle-aged population utilizing ultrasound and liver biopsy: a prospective study. Gastroenterology 2011;140:124-131.

4. Wong VW, Chu WC, Wong GL, Chan RS, Chim AM, Ong A, et al. Prevalence of non-alcoholic fatty liver disease and advanced fibrosis in Hong Kong Chinese: a population study using protonmagnetic resonance spectroscopy and transient elastography. Gut 2012;61:409-415.

5. Kwok R, Choi KC, Wong GL, Zhang Y, Chan HL, Luk AO, et al. Screening diabetic patients for non-alcoholic fatty liver disease with controlled attenuation parameter and liver stiffness measurements: a prospective cohort study. Gut 2015:309265.

6. O'Shea RS, Dasarathy S, McCullough AJ; Practice Guideline Committee of the American Association for the Study of Liver Diseases; Practice Parameters Committee of the American College of Gastroenterology. Alcoholic liver disease. Hepatology 2010;51:307-328.

7. Lozano R, Naghavi M, Foreman K, Lim S, Shibuya K, Aboyans V, et al. Global and regional mortality from 235 causes of death for 20 age groups in 1990 and 2010: a systematic analysis for the Global Burden of Disease Study 2010. Lancet 2012;380:2095-2128.

8. Lin DY, Sheen IS, Chiu CT, Lin SM, Kuo YC, Liaw YF. Ultrasonographic changes of early liver cirrhosis in chronic hepatitis B: a longitudinal study. J Clin Ultrasound 1993;21:303-308.

9. European Association for the Study of the Liver; Asociacion Latinoamericana para el Estudio del Higado. EASL-ALEH Clinical Practice Guidelines: Non-invasive tests for evaluation of liver disease severity and prognosis. J Hepatol 2015;63:237-264.

10. Mehta SH, Lau B, Afdhal NH, Thomas DL. Exceeding the limits of 
liver histology markers. J Hepatol 2009;50:36-41.

11. Angulo P, Hui JM, Marchesini G, Bugianesi E, George J, Farrell GC, et al. The NAFLD fibrosis score: a noninvasive system that identifies liver fibrosis in patients with NAFLD. Hepatology 2007;45:846-854.

12. Wong VW, Wong GL, Chim AM, Tse AM, Tsang SW, Hui AY, et al. Validation of the NAFLD fibrosis score in a Chinese population with low prevalence of advanced fibrosis. Am J Gastroenterol 2008;103:1682-1688.

13. Vergniol J, Boursier J, Coutzac C, Bertrais S, Foucher J, Angel C, et al. Evolution of noninvasive tests of liver fibrosis is associated with prognosis in patients with chronic hepatitis C. Hepatology 2014;60:65-76.

14. de Lédinghen V, Vergniol J, Barthe C, Foucher J, Chermak F, Le Bail B, et al. Non-invasive tests for fibrosis and liver stiffness predict 5 -year survival of patients chronically infected with hepatitis B virus. Aliment Pharmacol Ther 2013;37:979-988.

15. Wong VW, Chan HL. Transient elastography. J Gastroenterol Hepatol 2010;25:1726-1731.

16. Fraquelli M, Rigamonti C, Casazza G, Conte D, Donato MF, Ronchi G, et al. Reproducibility of transient elastography in the evaluation of liver fibrosis in patients with chronic liver disease. Gut 2007;56:968 973.

17. Wong VW, Vergniol J, Wong GL, Foucher J, Chan AW, Chermak F, et al. Liver stiffness measurement using XL probe in patients with nonalcoholic fatty liver disease. Am J Gastroenterol 2012;107:18621871.

18. Nguyen-Khac E, Chatelain D, Tramier B, Decrombecque C, Robert B, Joly JP, et al. Assessment of asymptomatic liver fibrosis in alcoholic patients using fibroscan: prospective comparison with seven noninvasive laboratory tests. Aliment Pharmacol Ther 2008;28:11881198.

19. Friedrich-Rust M, Wunder K, Kriener S, Sotoudeh F, Richter S, Bojunga J, et al. Liver fibrosis in viral hepatitis: noninvasive assessment with acoustic radiation force impulse imaging versus transient elastography. Radiology 2009;252:595-604.

20. Leung VY, Shen J, Wong VW, Abrigo J, Wong GL, Chim AM, et al. Quantitative elastography of liver fibrosis and spleen stiffness in chronic hepatitis B carriers: comparison of shear-wave elastography and transient elastography with liver biopsy correlation. Radiology 2013;269:910-918.

21. Loomba R, Wolfson T, Ang B, Hooker J, Behling C, Peterson M, et al. Magnetic resonance elastography predicts advanced fibrosis in patients with nonalcoholic fatty liver disease: a prospective study. Hepatology 2014;60:1920-1928.

22. Wong VW, Lampertico P, de Lédinghen V, Chang PE, Kim SU, Chen Y, et al. Probability-based interpretation of liver stiffness measurement in untreated chronic hepatitis B patients. Dig Dis Sci 2015;60:14481456.
23. Wong GL, Wong VW. Risk prediction of hepatitis B virus-related hepatocellular carcinoma in the era of antiviral therapy. World J Gastroenterol 2013;19:6515-6522.

24. Omata M, Lesmana LA, Tateishi R, Chen PJ, Lin SM, Yoshida H, et al. Asian Pacific Association for the Study of the Liver consensus recommendations on hepatocellular carcinoma. Hepatol Int 2010;4:439474.

25. Sherman M. Serological surveillance for hepatocellular carcinoma: time to quit. J Hepatol 2010;52:614-615.

26. Bruix J, Sherman M, American Association for the Study of Liver Diseases. Management of hepatocellular carcinoma: an update. Hepatology 2011;53:1020-1022.

27. Wong GL, Chan HL, Tse YK, Chan HY, Tse CH, Lo AO, et al. On-treatment alpha-fetoprotein is a specific tumor marker for hepatocellular carcinoma in patients with chronic hepatitis $B$ receiving entecavir. Hepatology 2014;59:986-995.

28. El-Serag HB. Hepatocellular carcinoma. N Engl J Med 2011;365:11181127.

29. Wong GL, Wong VW, Tan GM, Ip KI, Lai WK, Li YW, et al. Surveillance programme for hepatocellular carcinoma improves the survival of patients with chronic viral hepatitis. Liver Int 2008;28:79-87.

30. Jung KS, Kim SU, Ahn SH, Park YN, Kim do Y, Park JY, et al. Risk assessment of hepatitis B virus-related hepatocellular carcinoma development using liver stiffness measurement (FibroScan). Hepatology 2011;53:885-894.

31. Masuzaki R, Tateishi R, Yoshida H, Goto E, Sato T, Ohki T, et al. Prospective risk assessment for hepatocellular carcinoma development in patients with chronic hepatitis $\mathrm{C}$ by transient elastography. Hepatology 2009;49:1954-1961.

32. Shaheen AA, Wan AF, Myers RP. FibroTest and FibroScan for the prediction of hepatitis C-related fibrosis: a systematic review of diagnostic test accuracy. Am J Gastroenterol 2007;102:2589-2600.

33. Poynard T, Vergniol J, Ngo Y, Foucher J, Munteanu M, Merrouche W, et al. Staging chronic hepatitis $C$ in seven categories using fibrosis biomarker (FibroTest ${ }^{\mathrm{TM}}$ ) and transient elastography (FibroScan $®$ ). J Hepatol 2014;60:706-714.

34. Poynard T, Vergniol J, Ngo Y, Foucher J, Thibault V, Munteanu M, et al. Staging chronic hepatitis B into seven categories, defining inactive carriers and assessing treatment impact using a fibrosis biomarker (FibroTest ${ }^{\circledR}$ ) and elastography (FibroScan ${ }^{\circledR}$ ). J Hepatol 2014;61:994-1003.

35. Wong GL, Chan HL, Choi PC, Chan AW, Yu Z, Lai JW, et al. Noninvasive algorithm of enhanced liver fibrosis and liver stiffness measurement with transient elastography for advanced liver fibrosis in chronic hepatitis B. Aliment Pharmacol Ther 2014;39:197-208.

36. Kim BK, Kim HS, Yoo EJ, Oh EJ, Park JY, Kim do Y, et al. Risk assessment of clinical outcomes in Asian patients with chronic hepatitis B using enhanced liver fibrosis test. Hepatology 2014;60:1911-1919. 
37. Wong VW, Janssen HL. Can we use HCC risk scores to individualize surveillance in chronic hepatitis B infection? J Hepatol 2015;63:722732.

38. Wong GL, Chan HL, Wong CK, Leung C, Chan CY, Ho PP, et al. Liver stiffness-based optimization of hepatocellular carcinoma risk score in patients with chronic hepatitis B. J Hepatol 2014;60:339-345.

39. Wong VW, Chan SL, Mo F, Chan TC, Loong HH, Wong GL, et al. Clinical scoring system to predict hepatocellular carcinoma in chronic hepatitis B carriers. J Clin Oncol 2010;28:1660-1665.

40. Kim do Y, Song KJ, Kim SU, Yoo EJ, Park JY, Ahn SH, et al. Transient elastography-based risk estimation of hepatitis B virus-related occurrence of hepatocellular carcinoma: development and validation of a predictive model. Onco Targets Ther 2013;6:1463-1469.

41. Lee MH, Yang HI, Liu J, Batrla-Utermann $\mathrm{R}$, Jen $\mathrm{CL}$, Iloeje UH, et al. Prediction models of long-term cirrhosis and hepatocellular carcinoma risk in chronic hepatitis B patients: risk scores integrating host and virus profiles. Hepatology 2013;58:546-554.

42. Garcia-Tsao G, Groszmann RJ, Fisher RL, Conn HO, Atterbury CE, Glickman M. Portal pressure, presence of gastroesophageal varices and variceal bleeding. Hepatology 1985;5:419-424.

43. Garcia-Tsao G, Sanyal AJ, Grace ND, Carey W; Practice Guidelines Committee of the American Association for the Study of Liver Diseases; Practice Parameters Committee of the American College of Gastroenterology. Prevention and management of gastroesophageal varices and variceal hemorrhage in cirrhosis. Hepatology 2007:46:922-938.

44. Giannini E, Botta F, Borro P, Risso D, Romagnoli P, Fasoli A, et al. Platelet count/spleen diameter ratio: proposal and validation of a non-invasive parameter to predict the presence of oesophageal varices in patients with liver cirrhosis. Gut 2003:52:1200-1205.

45. Asrani SK, Kamath PS. Natural history of cirrhosis. Curr Gastroenterol Rep 2013;15:308.

46. Carrión JA, Navasa M, Bosch J, Bruguera M, Gilabert R, Forns X. Transient elastography for diagnosis of advanced fibrosis and portal hypertension in patients with hepatitis $C$ recurrence after liver transplantation. Liver Transpl 2006;12:1791-1798.

47. Lemoine M, Katsahian S, Ziol M, Nahon P, Ganne-Carrie N, Kazemi $F$, et al. Liver stiffness measurement as a predictive tool of clinically significant portal hypertension in patients with compensated hepatitis C virus or alcohol-related cirrhosis. Aliment Pharmacol Ther 2008:28:1102-1110.

48. Robic MA, Procopet B, Métivier S, Péron JM, Selves J, Vinel JP, et al. Liver stiffness accurately predicts portal hypertension related complications in patients with chronic liver disease: a prospective study. J Hepatol 2011;55:1017-1024.

49. Procopet B, Cristea VM, Robic MA, Grigorescu M, Agachi PS, Metivier $S$, et al. Serum tests, liver stiffness and artificial neural networks for diagnosing cirrhosis and portal hypertension. Dig Liver Dis
2015;47:411-416

50. Vizzutti F, Arena U, Romanelli RG, Rega L, Foschi M, Colagrande $S$, et al. Liver stiffness measurement predicts severe portal hypertension in patients with HCV-related cirrhosis. Hepatology 2007;45:1290-1297.

51. Colecchia A, Colli A, Casazza G, Mandolesi D, Schiumerini R, Reggiani $L B$, et al. Spleen stiffness measurement can predict clinical complications in compensated HCV-related cirrhosis: a prospective study. J Hepatol 2014;60:1158-1164.

52. Takuma Y, Nouso K, Morimoto Y, Tomokuni J, Sahara A, Toshikuni $N$, et al. Measurement of spleen stiffness by acoustic radiation force impulse imaging identifies cirrhotic patients with esophageal varices. Gastroenterology 2013;144:92-101. e2.

53. Tasu JP, Rocher $L, G P E$, Kuoch V, Kulh E, Miquel A, et al. Hepatic venous pressure gradients measured by duplex ultrasound. Clin Radiol 2002:57:746-752.

54. Choi YJ, Baik SK, Park DH, Kim MY, Kim HS, Lee DK, et al. Comparison of Doppler ultrasonography and the hepatic venous pressure gradient in assessing portal hypertension in liver cirrhosis. J Gastroenterol Hepatol 2003;18:424-429.

55. Taourel P, Blanc P, Dauzat M, Chabre M, Pradel J, Gallix B, et al. Doppler study of mesenteric, hepatic, and portal circulation in alcoholic cirrhosis: relationship between quantitative Doppler measurements and the severity of portal hypertension and hepatic failure. Hepatology 1998;28:932-936.

56. Berzigotti A, Casadei A, Magalotti D, Castaldini N, Losinno F, Rossi $C$, et al. Renovascular impedance correlates with portal pressure in patients with liver cirrhosis. Radiology 2006;240:581-586.

57. Vizzutti F, Arena U, Rega L, Romanelli RG, Colagrande S, Cuofano S, et al. Performance of Doppler ultrasound in the prediction of severe portal hypertension in hepatitis C virus-related chronic liver disease. Liver Int 2007;27:1379-1388.

58. Jeon SW, Cho CM, Tak WY, Ryeom HK, Kweon YO, Kim SK, et al. [The value of Doppler-ultrasonography and laboratory tests as noninvasive predictors of the presence of esophageal varices in patients with chronic liver disease]. Korean J Gastroenterol 2006:48:180-187.

59. Kim MY, Baik SK, Park DH, Lim DW, Kim JW, Kim HS, et al. Damping index of Doppler hepatic vein waveform to assess the severity of portal hypertension and response to propranolol in liver cirrhosis: a prospective nonrandomized study. Liver Int 2007;27:1103-1110.

60. Baik SK, Kim JW, Kim HS, Kwon SO, Kim YJ, Park JW, et al. Recent variceal bleeding: Doppler US hepatic vein waveform in assessment of severity of portal hypertension and vasoactive drug response. Radiology 2006;240:574-580.

61. Park SH, Park TE, Kim YM, Kim SJ, Baik GH, Kim JB, et al. Noninvasive model predicting clinically-significant portal hypertension in patients with advanced fibrosis. J Gastroenterol Hepatol 2009;24:1289-1293. 
62. Castéra L, Le Bail B, Roudot-Thoraval F, Bernard PH, Foucher J, Merrouche $W$, et al. Early detection in routine clinical practice of cirrhosis and oesophageal varices in chronic hepatitis C: comparison of transient elastography (FibroScan) with standard laboratory tests and non-invasive scores. J Hepatol 2009;50:59-68.

63. Vermehren J, Polta A, Zimmermann O, Herrmann E, Poynard T, Hofmann WP, et al. Comparison of acoustic radiation force impulse imaging with transient elastography for the detection of complications in patients with cirrhosis. Liver Int 2012;32:852-858.

64. Morishita N, Hiramatsu N, Oze T, Harada N, Yamada R, Miyazaki $M$, et al. Liver stiffness measurement by acoustic radiation force impulse is useful in predicting the presence of esophageal varices or high-risk esophageal varices among patients with HCV-related cirrhosis. J Gastroenterol 2014;49:1175-1182.

65. Mangone M, Moretti A, Alivernini F, Papi C, Orefice R, Dezi A, et al. Platelet count/spleen diameter ratio for non-invasive diagnosis of oesophageal varices: is it useful in compensated cirrhosis? Dig Liver Dis 2012;44:504-507.

66. Barrera F, Riquelme A, Soza A, Contreras A, Barrios G, Padilla O, et al. Platelet count/spleen diameter ratio for non-invasive prediction of high risk esophageal varices in cirrhotic patients. Ann Hepatol 2009:8:325-330.

67. Eslam M, Ampuero J, Jover M, Abd-Elhalim H, Rincon D, Shatat $M_{\text {, }}$ et al. Predicting portal hypertension and variceal bleeding using non-invasive measurements of metabolic variables. Ann Hepatol 2013;12:588-598.

68. Alcantara RV, Yamada RM, De Tommaso AM, Bellomo-Brandão $M A$, Hessel $G$. Non-invasive predictors of esophageous varices in children and adolescents with chronic liver disease or extrahepatic portal venous obstruction. J Pediatr (Rio J) 2012;88:341-346.

69. Cherian JV, Deepak N, Ponnusamy RP, Somasundaram A, Jayanthi V. Non-invasive predictors of esophageal varices. Saudi J Gastroenterol 2011;17:64-68.

70. Colecchia A, Di Biase AR, Scaioli E, Predieri B, lughetti L, Reggiani $M L$, et al. Non-invasive methods can predict oesophageal varices in patients with biliary atresia after a Kasai procedure. Dig Liver Dis 2011;43:659-663

71. de Franchis R; Baveno VI Faculty. Expanding consensus in portal hypertension: Report of the Baveno VI Consensus Workshop: Stratifying risk and individualizing care for portal hypertension. J Hepatol 2015;63:743-752.

72. Wong GL, Wong VW, Choi PC, Chan AW, Chim AM, Yiu KK, et al. On-treatment monitoring of liver fibrosis with transient elastography in chronic hepatitis B patients. Antivir Ther 2011;16:165-172.

73. Bolognesi M, Sacerdoti D, Merkel C, Bombonato G, Gatta A. Noninvasive grading of the severity of portal hypertension in cirrhotic patients by echo-color-Doppler. Ultrasound Med Biol 2001;27:901907.

74. Sebastiani G, Tempesta D, Fattovich G, Castera L, Halfon P, Bourliere $M$, et al. Prediction of oesophageal varices in hepatic cirrhosis by simple serum non-invasive markers: Results of a multicenter, largescale study. J Hepatol 2010;53:630-638.

75. Patanwala I, McMeekin P, Walters R, Mells G, Alexander G, Newton $J$, et al. A validated clinical tool for the prediction of varices in $\mathrm{PBC}$ : the Newcastle Varices in PBC Score. J Hepatol 2013;59:327-335.

76. Galal GM, Amin NF, Abdel Hafeez HA, El-Baz MA. Can serum fibrosis markers predict medium/large oesophageal varices in patients with liver cirrhosis? Arab J Gastroenterol 2011;12:62-67.

77. Hong WD, Zhu QH, Huang ZM, Chen XR, Jiang ZC, Xu SH, et al. Predictors of esophageal varices in patients with HBV-related cirrhosis: a retrospective study. BMC Gastroenterol 2009;5:9-11.

78. Thabut D, Trabut JB, Massard J, Rudler M, Muntenau M, Messous D, et al. Non-invasive diagnosis of large oesophageal varices with FibroTest in patients with cirrhosis: a preliminary retrospective study. Liver Int 2006;26:271-278.

79. Taourel P, Blanc P, Dauzat M, Chabre M, Pradel J, Gallix B, et al. Doppler study of mesenteric, hepatic, and portal circulation in alcoholic cirrhosis: relationship between quantitative Doppler measurements and the severity of portal hypertension and hepatic failure. Hepatology 1998;28:932-936. 